\title{
Intrinsic electron transport properties of carbon nanotube Y-junctions
}

\author{
Vincent Meunier ${ }^{\mathrm{a}}$ \\ Computer Science and Mathematics Division, Oak Ridge National Laboratory, Oak Ridge, Tennessee 37831
}

Marco Buongiorno Nardelli and J. Bernholc

Computer Science and Mathematics Division, Oak Ridge National Laboratory, Oak Ridge, Tennessee 37831

and Department of Physics, North Carolina State University, Raleigh, North Carolina 27695

Thomas Zacharia

Computing and Computational Sciences Directorate, Oak Ridge National Laboratory, Oak Ridge, Tennessee 37831

Jean-Christophe Charlier

Université Catholique de Louvain, PCPM \& CERMIN, B-1348 Louvain-La-Neuve, Belgium

(Received 16 September 2002; accepted 6 November 2002)

\begin{abstract}
The electron transport properties of three-terminal carbon-nanotube junctions are investigated within the Landauer theory of quantum conductance. Using a realistic tight-binding Hamiltonian, we demonstrate that the experimentally observed rectifying behavior is not an intrinsic property of the junction, but rather of the contact geometry. When semiconducting nanotubes are connected to metallic leads, nontransmitting states are induced at the nanotube-metal interface, leading to asymmetric transmission curves and potentially rectifying behavior of the nanodevice. (c) 2002 American Institute of Physics. [DOI: 10.1063/1.1533842]
\end{abstract}

Carbon nanotubes have attracted much interest since their early discovery, ${ }^{1}$ due to their peculiar electronic and geometrical properties. ${ }^{2}$ More recently, the possibility of forming seamless junctions between different carbon nanotubes has stimulated much modeling and experimental research. Seamless nanotube connections are realized via the introduction of topological defects (i.e., pentagons and heptagons) in the otherwise perfectly hexagonal lattice, while keeping the threefold coordination of each atom. ${ }^{3-5}$ Besides single or multiple connections between two different carbon nanotubes,${ }^{3-6}$ the possibility of connecting three, ${ }^{7,8}$ four, ${ }^{9}$ or more $^{10}$ nanotubes has increased the potential of these systems for use as active components in nanoscale electronic devices.

Three-terminal T- or Y-junctions have been observed in carbonaceous deposits formed during arc discharge growth as early as $1995 .{ }^{11}$ A controlled growth of large amounts of Y-junctions has been made possible using nanostructured templates. ${ }^{12}$ More recently, much work has been devoted to various methods of growing multiterminal systems, including three-terminal ${ }^{13-18}$ and up to five-terminal systems. ${ }^{10} \mathrm{An}$ other promising route to multiterminal nanotube systems is to directly connect individual carbon nanotubes using an electron beam welding technique. ${ }^{9}$ This procedure has the unique advantage of allowing the formation of single-walled multiterminal junctions. Turning to theory, models of multiterminal systems were proposed soon after the discovery of carbon nanotubes. ${ }^{7,8}$ Their stability has been theoretically confirmed, ${ }^{19}$ while their electronic and conductance properties were the focus of various studies. ${ }^{19-24}$

The key element that makes branched nanotube systems so potentially attractive has been experimentally demon- strated by Papadopoulous and coworkers, ${ }^{25}$ who performed electronic transport measurements on multiwalled Y-junctions and showed that they behave as intrinsic nonlinear devices, displaying strong rectifying behavior at room temperature. This finding elevated the three-terminal carbonnanotube systems to the rank of the ultimate singlemolecule-based transistor.

In a series of papers, ${ }^{21-23}$ Andriotis et al. theoretically investigated the electron transport properties of carbon nanotube Y-junctions. An almost perfect rectification behavior was calculated for semiconducting $(n, 0)$ symmetric systems coupled to metallic leads, independently of the angle between the branches of the junctions. In the present letter, we investigate the origin of the rectification and examine threeterminal junctions with and without metallic leads. Our results explicitly demonstrate the critical role played by the contacts in the rectification process, and show that a threeterminal geometry is not necessary for rectification.

The local density of states (LDOS) and coefficient of transmission are evaluated using a Green's function and transfer-matrix-based approach for computing transport in extended systems, ${ }^{26,27}$ generalized for multi-terminal transport. ${ }^{28}$ The method is applicable to any general Hamiltonian that can be described within a localized-orbital basis. In the present work, a Slater-Koster tight-binding Hamiltonian is used.

Consider a system consisting of $n$ terminals meeting at a branching region, referred hereafter as the "conductor" region. The current flowing from the $i$ th to the $j$ th terminal for a set of applied voltages $\widetilde{v}=\left\{V_{1} \ldots V_{n}\right\}$ is given by the Landauer formula ${ }^{29}$

$$
\begin{aligned}
I_{i j}(\widetilde{v})= & \frac{2 e^{2}}{h} \int T_{i j}(\widetilde{v}, E) \times\left[f_{0}\left(E-\mu_{i}-V_{i}\right)-f_{0}\left(E-\mu_{j}\right.\right. \\
& \left.\left.-V_{j}\right)\right] d E
\end{aligned}
$$






FIG. 1. (a) Electron transmission between any two terminals of the nanotube-terminated symmetric Y-junction, constructed from three $(10,0)$ nanotubes shown in the inset. (b) The same calculation for a perfect $(10,0)$ semiconducting nanotube. Note the different scales for the $y$-axis in the left and right panels.

where $f_{0}$ is the Fermi-Dirac distribution and $\mu_{i}$ is the chemical potential of terminal $i$. The two-terminal transmission is given by

$$
T_{i j}=\operatorname{Tr}\left(\Gamma_{i} G_{C}^{r} \Gamma_{j} G_{C}^{a}\right),
$$

and must be re-evaluated for each different set of applied bias $\widetilde{v}$. The coupling $\Gamma_{i}$ between the conductor and the $i$ th lead is expressed as a function of the advanced $(a)$ and retarded $(r)$ self-energies terms of the semi-infinite leads $\sum_{i}^{a, r}$ :

$$
\Gamma_{i}=i\left[\Sigma_{i}^{r}-\Sigma_{i}^{a}\right] \text {, }
$$

while the conductor Green's function $G_{C}$ is obtained from

$$
G_{C}=\left(\varepsilon-H_{C}-\sum_{i=1}^{n} \Sigma_{i}\right)^{-1} \text {, }
$$

where $\varepsilon=E+i \eta$ ( $\eta$ arbitrarily small) is a complex energy, and $H_{C}$ is the Hamiltonian matrix of the conductor region. In the present work, the self-energies are conveniently computed using the surface Green's function matching theory ${ }^{30}$ and an efficient, iterative transfer matrix procedure. ${ }^{26,27}$

In order to isolate the effect of the contacts with the metallic leads from the intrinsic properties of the junction, two separated systems are considered. In both systems, the central part is made of a Y-junction composed of three finite $(10,0)$ nanotube branches [Fig. 1(a), inset]. In system I, the nanotube branches are seamlessly extended by three semiinfinite $(10,0)$ nanotubes, acting as leads. In system II, the leads consist of semi-infinite metallic electrodes $(\mathrm{Ni})$, as in Ref. 22. The corresponding coefficients of transmission through any two of the three branches are plotted in Figs. 1 and 2, left panels. As a reference, the same calculations are performed on a single $(10,0)$ carbon nanotube (Figs. 1 and 2, right panels). The transmission of electrons through system I looks much like the one of a pure $(10,0)$ nanotube, except for a factor of about 2, which is due to the almost even splitting between the branches. ${ }^{31}$ No important asymmetry is observed, as expected for undoped semiconductors.

However, the metal-terminated Y-junction displays a strongly asymmetric conductance curve [Fig. 2(a)]. Note that the same effect is also observed in the metal-terminated perfect nanotube [Fig. 2(b)], which demonstrates that the rectification originates from the presence of interfaces between the "nanodevice" and the metallic leads, as shown subsequently.

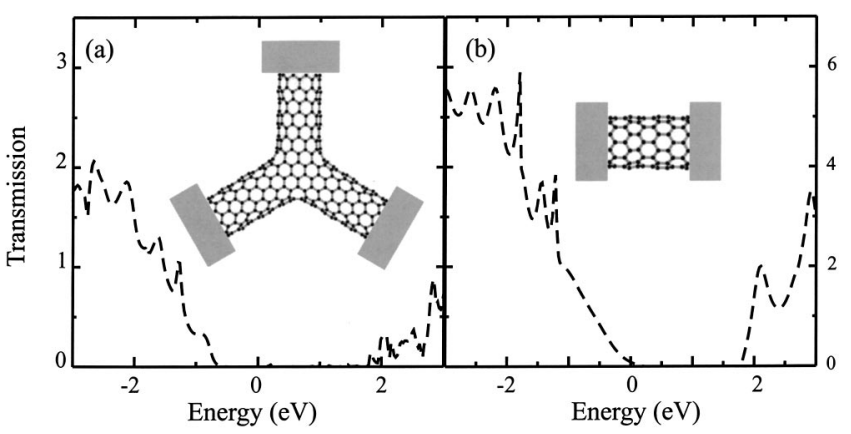

FIG. 2. (a) Electron transmission between any two terminals of a metalterminated symmetric Y-junction, constructed from three $(10,0)$ nanotubes shown in the inset. (b) The same calculation for a perfect $(10,0)$ semiconducting nanotube. Note the different scales for the $y$-axis in the left and right panels.

Although Fig. 1 shows conclusively that a perfect nanotube-terminated junction does not display a rectifying behavior, it is important to understand the origin of the asymmetry that appears in the transmission curves when the system is connected to metallic leads. To this end, LDOS along both Y-systems have been computed and plotted in Fig. 3. The curves represented in Fig. 3 are averaged over circular sections perpendicular to the axes of the branches, typically over 20 sites, except at the joint, where the number of atoms increases to 26. The averaged LDOS of System I [Fig. 3(a)] resembles the one of an infinite $(10,0)$ nanotube, aside from resonance donor peaks associated with the six heptagons located at the joint. The intensity of the resonance peaks decreases rapidly along each arm of the junction, when moving from the center of the junction (top) towards the semi-infinite terminal (bottom). For the metal-junction interface, however, a large number of peaks are observed in the positive energy window [Fig. 3(b)]. These states can be regarded as scattering states, which correspond to nontransmitting elec-
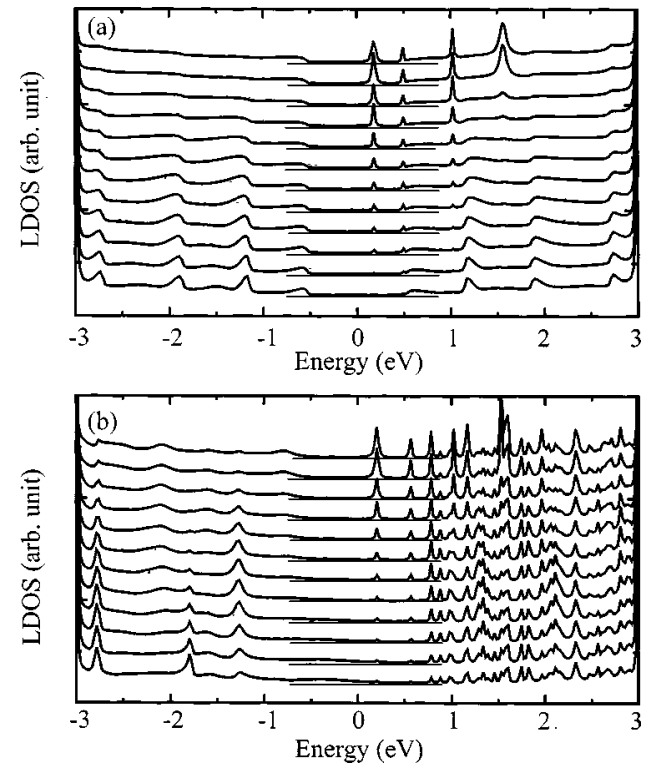

FIG. 3. Averaged LDOS along one branch of the $3 \times(10,0)$ Y-nanotube from the branching (top) to the nanotube-lead interface (bottom). Panels (a) and (b) correspond to the pure carbon and the metal-terminated systems, respectively. The origin at the energy scale coincides with the Fermi energy of the conductor. The horizontal lines correspond to the zero density of states for each curve. 
trons. The transmission of electrons through the interface is therefore hindered for positive energy values, leading to a drastic reduction of the conductance. Since these scattering states only affect positive energy values while leaving the negative window basically untouched, they lead to potentially asymmetric $I-V$ characteristics. ${ }^{32}$

Our results are consistent with previous theoretical investigations of carbon nanotube devices, which can be understood in terms of the formation of Schottky barriers at the interfaces of nanotube heterojunctions, provided that doping is present in the system. ${ }^{33,34}$ Furthermore, it has been shown recently by Heinze et al. ${ }^{35}$ that carbon-nanotube-based fieldeffect transistors operate as "Schottky barrier transistors," in which modulation of resistance at the contact plays the primary role in switching, rather than the channel conductance.

One should mention that a completely quantitative description of the interface between the nanotube channel and the metallic leads requires a fully self-consistent approach, which takes into account charge transfer and band alignment, ${ }^{36}$ and that only qualitative information concerning the lead-channel interface can be obtained within the current non-self-consistent approach.

In summary, we have shown that the rectifying behavior of carbon nanotube Y-junctions is not an intrinsic property of the branching, but is solely due to the properties of the interfaces between the nanotube branches and the metallic leads. The rectification arises from scattering by the interfacial states, which are closely related to the creation of Schottky barriers at the metal-nanotube contacts. The magnitude of the Schottky barrier strongly depends upon the detailed atomic geometry of the contact. An intrinsic, carbonnanotube-terminated Y-junction does not display an asymmetric conductance spectrum, but behaves as a junction between undoped semiconductors. Similar behaviors are expected for any three-terminal carbon-nanotube systems, including nonsymmetric $\mathrm{Y}$ - and T-junctions.

This research is sponsored in part by the Mathematical, Information and Computational Sciences Division, Office of Advanced Scientific Computing Reasearch of the U.S. Department of Energy under Contract No. DE-AC0500OR22725 with UT-Battelle, LLC. One of the authors (J. B.) was supported in part by DOE Grant No. DE-FG0298ER45685. Two of the authors (V.M. and J.C.C.) acknowledge the National Fund for Scientific Research (FNRS) of Belgium. Part of this work presents research results of the Belgian Program on Inter-university Attraction Poles (PAI5/ 1/1) on Quantum Size Effects in Nano-structured Materials.

\footnotetext{
${ }^{1}$ S. Iijima, Nature (London) 354, 56 (1991).

${ }^{2}$ For a recent review of the electronic and structural properties of carbon
}

nanotubes, see for example: J. Bernholc, D. Brenner, M. Buongiorno Nardelli, V. Meunier, and C. Roland, Annu. Rev. Mater. Res. 32, 347 (2002).

${ }^{3}$ B. I. Dunlap, Phys. Rev. B 50, 8134 (1994).

${ }^{4}$ P. Lambin, A. Fonseca, J. P. Vigneron, J. B. Nagy, and A. A. Lucas, Chem. Phys. Lett. 245, 85 (1995).

${ }^{5}$ L. Chico, V. H. Crespi, L. X. Benedict, S. G. Louie, and M. L. Cohen, Phys. Rev. Lett. 76, 971 (1996).

${ }^{6}$ V. Meunier, M. Buongiorno Nardelli, C. Roland, and J. Bernholc, Phys. Rev. B 64, 195419 (2001).

${ }^{7}$ L. Chernozatonskii, Phys. Lett. A 172, 173 (1992).

${ }^{8}$ G. E. Scuseria, Chem. Phys. Lett. 195, 534 (1992).

${ }^{9}$ M. Terrones, F. Banhart, N. Grobert, J.-C. Charlier, H. Terrones, and P. M. Ajayan, Phys. Rev. Lett. 89, 075505 (2002).

${ }^{10}$ J. M. Ting and C. C. Chang, Appl. Phys. Lett. 80, 324 (2002).

${ }^{11}$ D. Zhou and S. Seraphin, Chem. Phys. Lett. 238, 286 (1995).

${ }^{12}$ J. Li, C. Papadopoulos, and J. Xu, Nature (London) 402, 253 (1999).

${ }^{13}$ B. Gan, J. Ahn, Q. Zhang, Q. F. Huang, C. Kerlit, S. F. Yoon, Rusli, V. A. Ligachev, X. B. Zhang, and W. Z. Li, Mater. Lett. 45, 315 (2000).

${ }^{14}$ B. C. Satishkumar, P. J. Thomas, A. Govindaraj, and C. N. R. Rao, Appl. Phys. Lett. 77, 2530 (2000).

${ }^{15}$ B. Gan, J. Ahn, Q. Zhang, Rusli, S. F. Yoon, J. Yu, Q. F. Huang, K. Chew, V. A. Ligatchev, X. B. Zhang, and W. Z. Li, Chem. Phys. Lett. 333, 23 (2001)

${ }^{16}$ F. L. Deepak, A. Govindaraj, and C. N. R. Rao, Chem. Phys. Lett. 345, 5 (2001).

${ }^{17}$ T. Gao, G. Meng, J. Zhang, S. Sun, and L. Zhang, Appl. Phys. A: Mater. Sci. Process. 74, 403 (2002).

${ }^{18}$ H. W. Zhu, L. J. Ci, C. L. Xu, J. Liang, and D. H. Wu, Diamond Relat. Mater. 11, 1349 (2002).

${ }^{19}$ M. Menon and D. Srivastava, Phys. Rev. Lett. 79, 4453 (1997).

${ }^{20}$ G. Treboux, P. Lapstun, and K. Siiverbrook, Chem. Phys. Lett. 306, 402 (1999).

${ }^{21}$ A. N. Andriotis, M. Menon, D. Srivastava, and L. Chernozatonskii, Appl. Phys. Lett. 79, 266 (2001).

${ }^{22}$ A. N. Andriotis, M. Menon, D. Srivastava, and L. Chernozatonskii, Phys. Rev. Lett. 8706, 066802 (2001).

${ }^{23}$ A. N. Andriotis, M. Menon, D. Srivastava, and L. Chernozatonskii, Phys. Rev. B 65, 165416 (2002).

${ }^{24}$ A. Peres-Garrido and A. Urbina, Carbon 40, 1227 (2002).

${ }^{25}$ C. Papadopoulos, A. Rakitin, J. Li, A. S. Vedeneev, and J. M. Xu, Phys. Rev. Lett. 85, 3476 (2000).

${ }^{26}$ M. Buongiono Nardelli and J. Bernholc, Phys. Rev. B 60, R16338 (1999).

${ }^{27}$ M. Buongiono Nardelli, Phys. Rev. B 60, 7828 (1999).

${ }^{28}$ V. Meunier and M. Buongiorno Nardelli, (unpublished).

${ }^{29}$ R. Landauer, IBM J. Res. Dev. 32, 306 (1988).

${ }^{30}$ F. Garcia-Moliner and V. Velasco, Phys. Rep. 200, 83 (1991).

${ }^{31}$ Even though the system has a perfect threefold symmetry, the transmission is not exactly divided by two, since part of the electron wave function is reflected on the conductor, in particular on the heptagons. The presence of the heptagons is also responsible for the appearance of a small asymmetry in the conductance, compared to a pristine nanotube.

${ }^{32}$ Rectifying behavior will be induced by asymmetric transmission curves, provided that an asymmetric potential profile breaks the geometrical symmetry of the device. For a detailed discussion of the effect of the potential drop see: W. Tian, S. Datta, S. Hong, R. Reifenberger, J. I. Henderson, and C. P. Kubiak, J. Chem. Phys. 109, 2874 (1998).

${ }^{33}$ F. Leonard and J. Tersoff, Phys. Rev. Lett. 84, 4693 (2000).

${ }^{34}$ A. A. Odintsov, Phys. Rev. Lett. 85, 150 (2000).

${ }^{35}$ S. Heinze, J. Tersoff, R. Martel, V. Derijcke, J. Appenzeller, and P. Avouris, Phys. Rev. Lett. 89, 106801 (2002).

${ }^{36}$ M. Buongiorno Nardelli, J.-L. Fattebert, and J. Bernholc, Phys. Rev. B 64, 245423 (2001). 